\title{
Osteosarcoma of Rib Treated with Regorafenib: A Case Report
}

Orkun SAKAR ${ }^{1}$, Cemre UÇARYILMAZ², Ahmet Bilgehan ŞAHIN ${ }^{3}$ Türkkan EVRENSEL ${ }^{3}$, Cengiz GEBİTEKİN $^{4}$

${ }^{1}$ Bursa Uludağ University Faculty of Medicine, Department of Internal Medicine, Bursa, Turkey

${ }^{2}$ Koç University, Institute of Health Sciences, Istanbul, Turkey

${ }^{3}$ Bursa Uludağ University Faculty of Medicine, Division of Medical Oncology, Bursa, Turkey

${ }^{4}$ Bursa Uludağ University Faculty of Medicine, Department of Thoracic Surgery, Bursa, Turkey

\begin{abstract}
Osteosarcoma is a common primary malignant bone tumor in young adults presenting with back and chest pain complaints. Here, we presented a rare case of osteosarcoma.
\end{abstract}

Keywords: Oncology, osteosarcoma of rib, regorafenib

\section{Introduction}

Osteosarcoma is the most common primary malignant bone tumor among young adults. ${ }^{1}$ It is more frequently seen in men than women. It usually originates from the metaphysis of long bones. Primary osteosarcoma rarely occurs in the rib. Modalities such as surgery, chemotherapy, and radiotherapy form the basis of the treatment, which should be planned in a multidisciplinary way. In this report, a case of osteosarcoma originating from the rib and spreading to the cervical and thoracic vertebrae is discussed.

\section{Case Report}

A 23-year-old female patient was admitted to our center with left-back pain for almost a year. In thoracic computed tomography (CT), a mass originating from bone with mixed density, causing erosion in the cortex, and covering the entire posterior and lateral arches and most of the anterior arch of the 1st left rib was detected (Figure 1). The mass at the level of T1 vertebra was compressing the left lung apex. The 1st rib, expanded by the mass, was completely removed by dissection. Resected material was reported as $8 \times 8 \mathrm{~cm}$ osteoblastic type osteosarcoma and surgical margins were

Received:February 07,2021;Accepted:March 3,2021; Published Online:March 6, 2021 


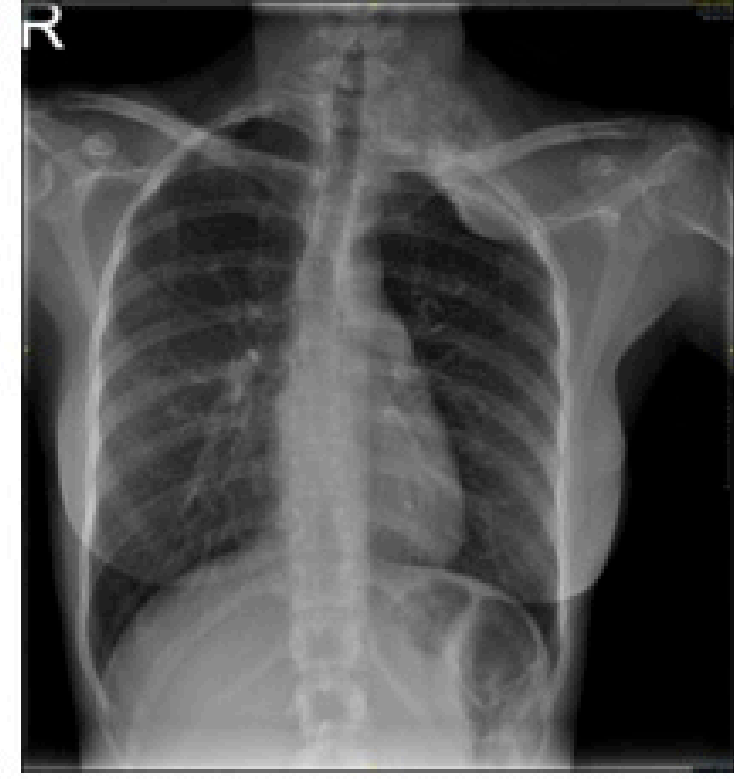

Figure 1. X-ray graph before diagnostic surgery.
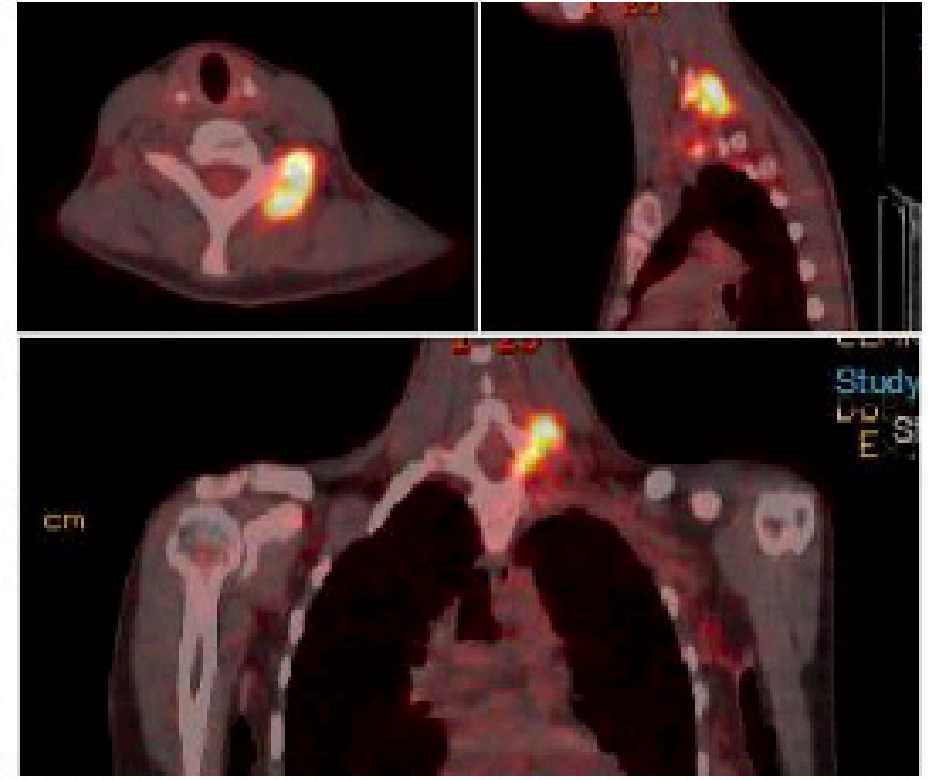

Figure 2. Systemic evaluation after diagnostic surgery.
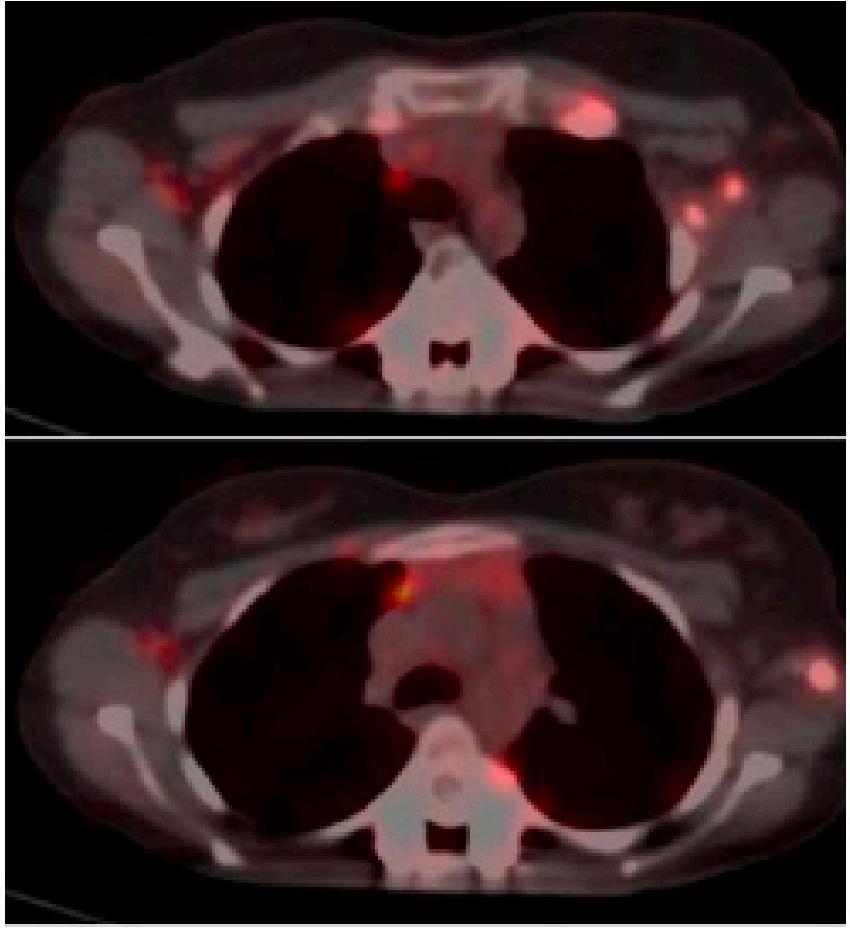

Figure 3. Recurrence at left axilla and left $1^{\text {st }}$ costochondral junction in PET-CT.

positive. The PET-CT for systemic evaluation showed a hypermetabolic mass (Suv-max 12-16) of $27 \times 28 \mathrm{~mm}$ in the left half of the T1 vertebra corpus and the left transverse process of the $\mathrm{C} 7$ vertebra (Figure 2). Thereon, the patient was treated with doxorubicin and cisplatin. The PET-CT following the third cycle of chemotherapy demonstrated a partial response, and the patient was evaluated as

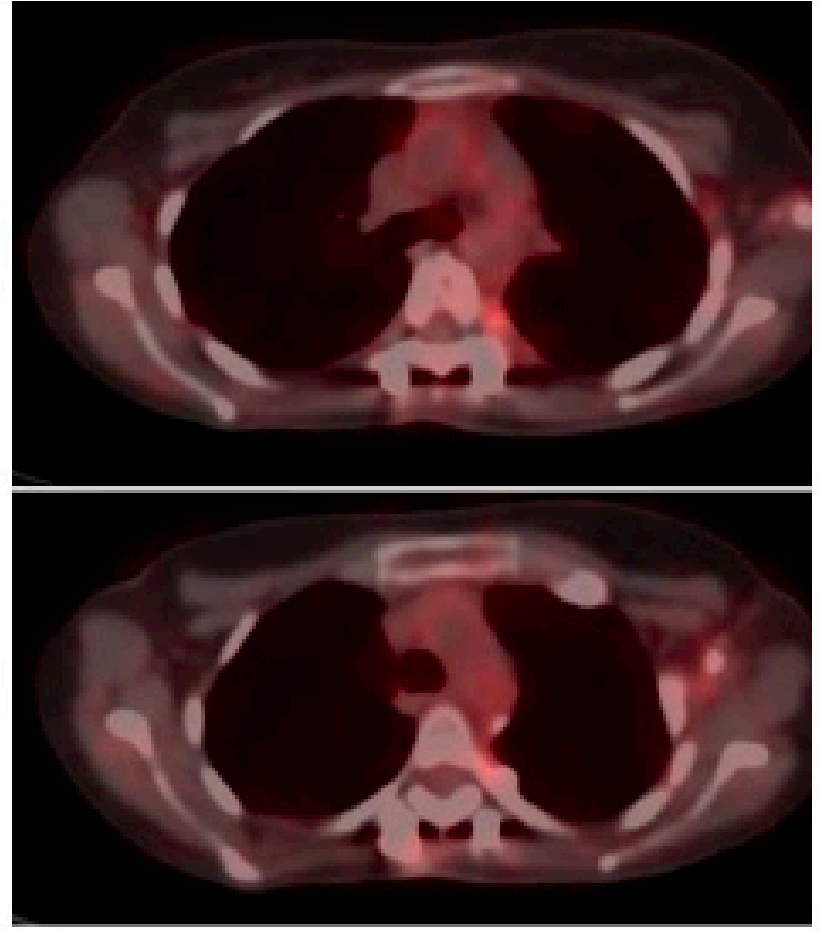

Figure 4. PET-CT images after local radiotherapy and regorafenib treatment.

resectable. All of the $\mathrm{C} 7, \mathrm{~T} 1$, and $\mathrm{T} 2$ vertebrae and the posterior arch of the 2 nd left rib were removed with en bloc resection. The mass was reported as $6 \times 4.5 \mathrm{~cm}$ osteosarcoma. Surgical margins were positive, and necrosis was $10 \%$. Radiotherapy was planned for the patient since the margins were positive, with the low chemotherapy response, interpreted as grade 1. 45 Gy radiotherapy was 
administered to the cervicodorsal region in 25 fractions. The chemotherapy regimen was changed, and docetaxel and gemcitabine were started. During the follow-up after adjuvant chemotherapy, a nodular lesion was detected in the left axilla on CT in the 4th month, and the biopsy was compatible with recurrence. PET-CT scan showed increased metabolic activities within nodular lesions in the left axilla and 1st left costochondral junction (Figure 3). Regorafenib was started after local radiotherapy. The patient continues to be followed up in the tenth month under regorafenib treatment (Figure 4).

\section{Discussion}

Osteosarcoma is the most common malignant tumor with the origin of bone in adults. Although malignant bone tumors located in the chest wall are most commonly originating from the ribs, primary osteosarcoma of the ribs is extremely rare, its prognosis is worse than osteosarcoma from long bones and there is a limited number of cases in the literature. Back and chest pain is the most important factors for clinical presentation. En bloc resection of the mass and the negative surgical margins are the main factors determining the prognosis of the disease. Survival is aimed to be increased with neoadjuvant and adjuvant chemotherapy. Radiotherapy may be considered in patients with positive surgical margins after resection of large masses. There are various studies showing the efficacy of cytotoxic therapy with regorafenib in recurrent cases. ${ }^{2,3}$ In patients presented with back and chest pain, there may be delays during the diagnosis of osteosarcoma of the rib because there are other reasons to be considered first. In the patient discussed in this report, the tumor has already reached large dimensions at the time of diagnosis because the clinical finding was only pain. Upon surgery, postoperative surgical margins were positive, and she experienced progression despite cytotoxic chemotherapy and radiotherapy. With regorafenib which is started later, the disease was controlled with partial response.

In conclusion, osteosarcoma of the rib is rare, and recurrence is common. Survival can be prolonged with a multidisciplinary approach including surgery, systemic chemotherapy, targeted agents, and radiotherapy.

\section{Conflict of Interests}

Authors declare that there are none.

\section{Acknowledgment}

This study has been presented in $17^{\text {th }}$ Uludag Internal Medicine National Winter Congress, $6^{\text {th }}$ Bursa Family Medicine Association National Congress, $11^{\text {th }}$ Uludag Internal Medicine Nursing Congress, 5-7 March 2021, Bursa, Turkey.

\section{References}

1. Mirabello L, Troisi R J, Savage S A. Osteosarcoma incidence and survival rates from 1973 to 2004: data from the Surveillance, Epidemiology, and End Results Program. Cancer. 2009 Apr 1;115(7):1531-43. doi: 10.1002/cncr.24121.

2. Davis L E, Bolejack V, Ryan C W, Ganjoo K N, Loggers E T, Chawla S, Agulnik M, Livingston M B, Reed D, Keedy V, Rushing D, Okuno S, Reinke D K, Riedel R F, Attia S, Mascarenhas L, Maki R G. Randomized Double-Blind Phase II Study of Regorafenib in Patients With Metastatic Osteosarcoma. J Clin Oncol . 2019 Jun 1;37(16):1424-31. doi: 10.1200/JCO.18.02374.

3. Duffaud F, Mir O, Boudou-Rouquette P, Piperno-Neumann S, Penel N, Bompas E, Delcambre C, Kalbacher E, Italiano A, Collard O, Chevreau C, Saada E, Isambert N, Delaye J, Schiffler C, Bouvier C, Vidal V, Chabaud S, Blay JY, French Sarcoma Group. Efficacy and safety of regorafenib in adult patients with metastatic osteosarcoma: a non-comparative, randomised, double-blind, placebo-controlled, phase 2 study. Lancet Oncol. 2019 Jan;20(1):120-33. doi: 10.1016/S14702045(18)30742-3. 